\title{
Effect of Phosphate Buffered Saline Solutions on Top-Down Fabricated ZnO Nanowire Field Effect Transistor
}

\author{
S. M. Sultan, ${ }^{1}$ M. R. R. de Planque, ${ }^{2}$ P. Ashburn, ${ }^{2}$ and H. M. H. Chong ${ }^{2}$ \\ ${ }^{1}$ Faculty of Electrical Engineering, Universiti Teknologi Malaysia, 81310 Skudai, Johor, Malaysia \\ ${ }^{2}$ University of Southampton, Southampton SO17 1BJ, UK \\ Correspondence should be addressed to S. M. Sultan; suhanasultan@utm.my
}

Received 7 August 2017; Revised 26 October 2017; Accepted 13 November 2017; Published 28 December 2017

Academic Editor: Surinder Singh

Copyright (C) 2017 S. M. Sultan et al. This is an open access article distributed under the Creative Commons Attribution License, which permits unrestricted use, distribution, and reproduction in any medium, provided the original work is properly cited.

ZnO based nanowire FETs have been fabricated by implementing a top-down approach, which uses optical photolithography, atomic layer deposition (ALD) of $\mathrm{ZnO}$ thin film, and anisotropic plasma etching. The effects of Phosphate Buffered Saline (PBS) solution on the surface of $\mathrm{ZnO}$ nanowire were investigated by measuring the FET characteristics at different PBS dilutions. The drain current, $I_{\mathrm{ON}}$, exhibited an increase of 39 times in the highest PBS solution concentration compared to measurement in air. From the measured transfer characteristics and output characteristics in various PBS dilutions, the device was found to maintain n-type behaviour. These results indicate that the device can be effectively used for biomolecules sensing.

\section{Introduction}

In the last two decades, nanomaterials including nanotubes, nanoscale films, nanogaps, nanoparticles, and quantum dots [1-6] have garnered vast consideration owing to their apt characteristics for constructing innovative nanoscale biosensors. For instance, size of nanomaterials in the range of one to $100 \mathrm{~nm}$ is suitable to be employed in cellular biology studies encompassing viruses, nucleic acid, cells, and proteins. Due to their diminutive size, nanomaterials have been developed to monitor and regulate noteworthy fields including, drug manufacturing, environment protection, food manufacturing, medical diagnosis, and toxicology screening [7]. Additionally, the great surface-to-volume ratio for nanomaterials enables a significant quantity of biomaterials-associated atoms to be positioned either at or near to their surface area.

Up until now, a variety of studies in major fields of biology have applied various methods of nanoscale sensing. Particularly in observing biological entities, a swift and accurate detection is critical. Attributed to this, the need for sensor-based system architectures becomes more demanding [3]. Such system architectures need to emphasize specificity, sensitivity, and cost involved in innovating and manufacturing nanoscale biosensors. In the field of electrical biosensing, field-effect transistors (FETs) devices are among the most heavily focused biosensors owing to their capability in direct translation of target biomolecules-FET surface interactions into intelligible electrical signals $[2,3,6,8-10]$.

Biosensing devices that are based on FETs possess a channel that contacts with the surroundings directly, which subsequently provides a greater surface charge control. Attributed to this, FET-based nanowires have high sensitivity. Nanowires are sensitive to changes in biological activities whereby their semiconductor channel surface picks up disparity in surface potential, which in turn modulates conductance of the channel. A number of reports have reported the achievements of biosensing devices with ultra-sensitivity manufactured from FETs based on nanomaterials encompassing single-walled carbon nanotubes (SWNT) [11], graphene [12], and metal oxide NWFETs [13-16].

Zinc oxide $(\mathrm{ZnO})$ is widely researched compounds to be used as a nanomaterial, owing to its vast prospect applications. The structure of this nanomaterial is known to exhibit great biocompatibility and great surface area, being nontoxic and chemically stable as well as exhibit high electron interaction characteristics and biomimicry, resulting in its suitability to be utilized as a biosensor [17]. Various literatures have studied the nanostructures of $\mathrm{ZnO}$ for biosensing technology, through a bottom-up approach [16-19]. Bottomup fabricated nanowire sensors commonly involve a complex 


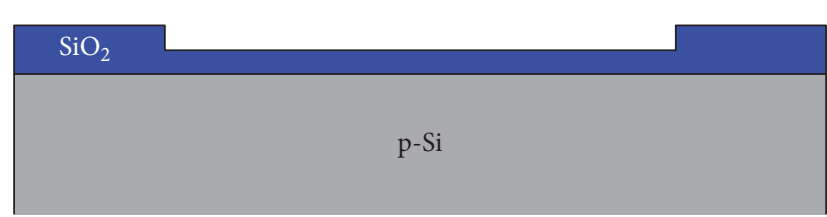

(a)

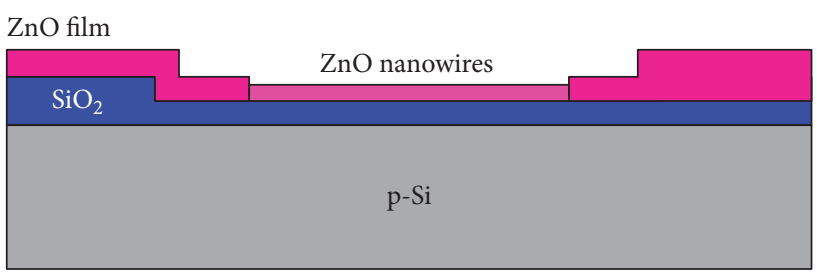

(c)

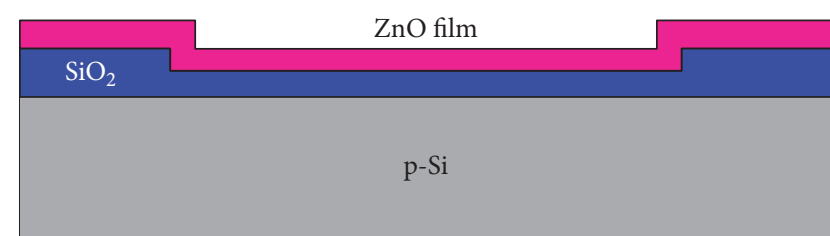

(b)

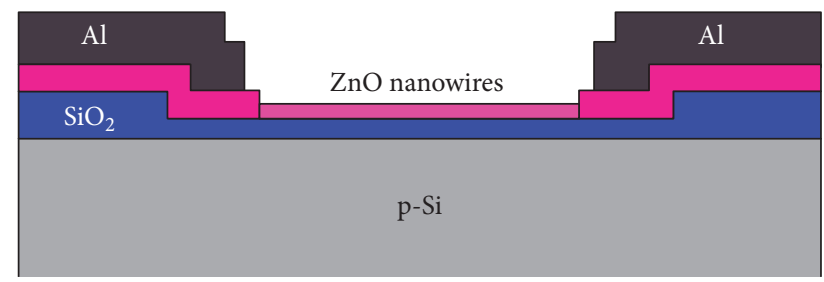

(d)

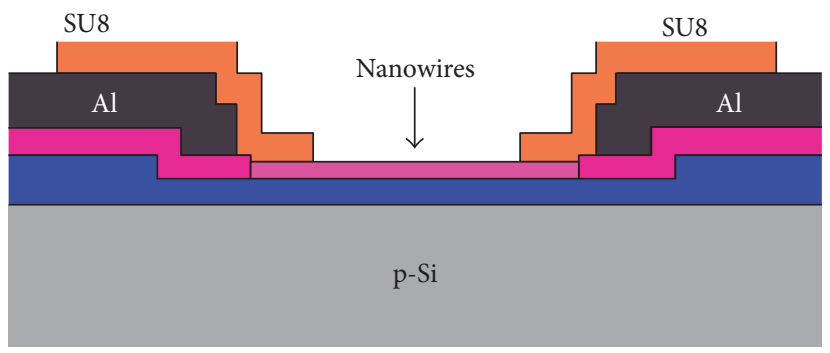

(e)

Figure 1: Illustrated process flow of $\mathrm{ZnO}$ nanowire biosensor: (a) $\mathrm{SiO}_{2}$ grown and etched on p-Si, (b) $\mathrm{ZnO}$ film deposited in ALD, (c) $\mathrm{ZnO}$ etched using spacer technique, and (d) Al contacts were sputtered and (e) SU-8 photoresist was coated and patterned.

fabrication process because it requires a single strand of semiconducting nanowire to be manipulated and aligned between the source and drain. Hence, repeatability is a challenge during fabrication of devices for large sensor arrays [20]. Therefore, developing an economically feasible and modest fabrication in large-scale manufacturing of sensors based on nanowires would speed up their acceptance as point-of-care (PoC) devices [21].

Previous works $[13,22]$ have demonstrated manufactured economically feasible nanowires in a regulated top-down fabricating approach. Since biosensing devices are normally operated in a liquid environment, the buffer concentration of the electrolyte can have critical effects on the sensing performances. In addition, the ion concentration in Phosphate Buffered Saline (PBS) matches that of the fluid inside the cells. In tandem with previous works [13, 22], this work explores electrical conductance modulation of nanowire FETs that have been manufactured via top-down approach in different concentration of Phosphate Buffered Saline (PBS) solutions.

\section{Experiment}

2.1. Fabrication. The fabrication process of $\mathrm{ZnO}$ nanowire FET is shown in Figure 1. A thermal silicon dioxide of about $200 \mathrm{~nm}$ was grown on low resistive $p$-Si wafers (Figure 1(a)). Then, the $\mathrm{SiO}_{2}$ layer was patterned using photolithography and subsequently etched in Reactive Ion Etch (RIE) for $100 \mathrm{~nm}$ to form $\mathrm{SiO}_{2}$ trenches or spacers (Figure 1(a)). Before thin film deposition, Fuming Nitric Acid was used to clean the samples to remove the photoresist. After cleaning, samples were immediately loaded into the atomic layer deposition (ALD) chamber for $\mathrm{ZnO}$ film deposition (Figure 1(b)). $\mathrm{ZnO}$ film was deposited at a temperature of $100^{\circ} \mathrm{C}$ with the DEZ precursor dose time and $\mathrm{O}_{2}$ plasma time set at $30 \mathrm{~ms}$ and $4 \mathrm{~s}$, respectively

After the $\mathrm{ZnO}$ thin film ALD deposition, the samples were patterned by photolithography and etched anisotropically in Inductively Coupled Plasma (ICP) to form array of nanowires (Figure 1(c)). Subsequently, the samples were patterned by photolithography for $\mathrm{Al}$ deposition. A $200 \mathrm{~nm}$ $\mathrm{Al}$ was evaporated before the $\mathrm{Al}$ lift-off process (Figure 1(d)) was executed in NMP solution. After metallization process, $4.8 \mu \mathrm{m}$ thickness of SU8-3005 photoresist was spincoated. Using photolithography, the resist was patterned and developed to open the sensing windows as illustrated in Figure 1(e).

The bio-FET device fabricated consists of an array of 100 nanowires with channel length of $20 \mu \mathrm{m}$ [20]. The electrical characteristics measurements are executed at room temperature and in dark natural ambient using Agilent 15000 Parameter Analyzer.

2.2. PBS Measurements. Figure 2(a) shows the scanning electron microscope (SEM) image of fabricated $\mathrm{ZnO}$ nanowires after the ICP etch. A pair of $\mathrm{ZnO}$ nanowires was clearly visible along the $\mathrm{SiO}_{2}$ trench. Figure 2(b) shows the optical image 


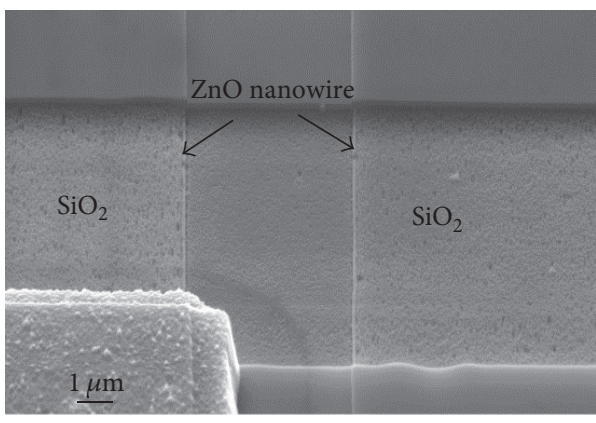

(a)

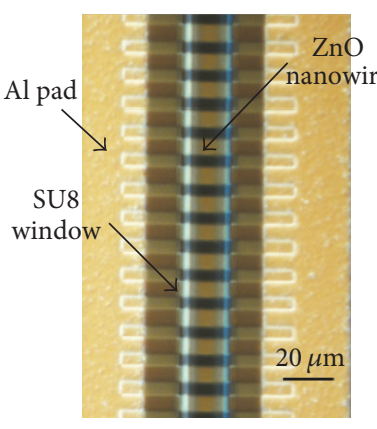

(b)

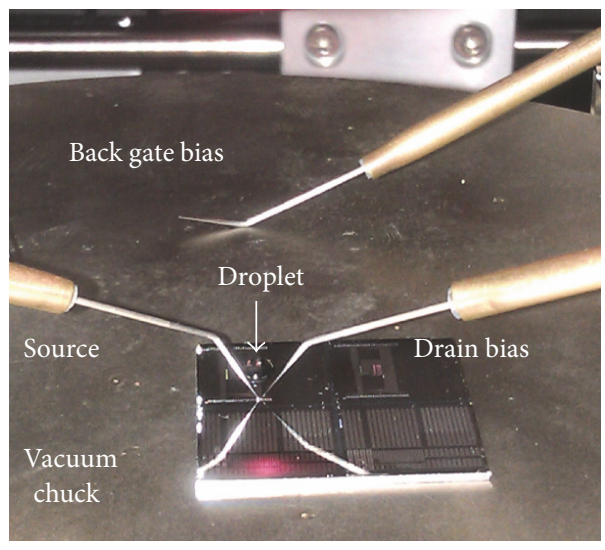

(c)

Figure 2: (a) SEM image of ZnO nanowires after ICP etch. (b) Optical image of a completed nanowire biosensor. (c) Biosensing measurement setup.

of fabricated biosensor. There is an opening window with length of $20 \mu \mathrm{m}$ which is used to expose the nanowires to the solution. There are offsets which are measured to be $3.4 \mu \mathrm{m}$ and $2.3 \mu \mathrm{m}$ between the SU8 window edges and the nearest source/drain, respectively. These offsets ensure that the source and drain are not connected through the liquid when the sensor is exposed to various solutions [20].

The measurements started by introducing $5 \mu \mathrm{L}$ aqueous droplet on the nanowire surface. It is important to ensure that the solution, which was introduced, covers the nanowire window as shown in Figure 2(b). The transfer characteristics and the output characteristics were measured within 10 secs after the solution was dropped on the nanowire surface. After each measurement was taken, the surface of the nanowire was cleaned by rinsing with a drop of deionized water and dried the surface with tissue. This step was repeated for ten times to confirm that the surface on the nanowire is completely dried before a new droplet is introduced.

The PBS solutions were diluted in deionized water with $\times 1000, \times 100$, and $\times 10$ dilutions. A PBS solution with $100 \mathrm{ml}$ deionized water contains $137 \mathrm{mM}$ of $\mathrm{NaCl}, 2.7 \mathrm{mM}$ of $\mathrm{KCl}$, $8.1 \mathrm{mM}$ of $\mathrm{Na}_{2} \mathrm{HPO}_{4}, 1.5 \mathrm{mM}$ of $\mathrm{KH}_{2} \mathrm{PO}_{4}, 140 \mathrm{mM}$ of salt, and $10 \mathrm{mM}$ buffer with $\mathrm{pH}$ 7.4. A PBS solution with $\times 1000, \times 100$, and $\times 10$ dilutions contains $0.14 \mathrm{mM}, 1.4 \mathrm{mM}$, and $14 \mathrm{mM}$ of salt at $\mathrm{pH}$ 7.4, respectively. Electrical characteristics were measured in each PBS dilution.

The biosensing measurements were executed following these orders: (i) measurement in air; (ii) measurement in PBS $\times 1000$ dilutions; (iii) measurement in air; (iv) measurement in PBS $\times 100$ dilutions; (v) measurement in air; (vi) measurement in PBS $\times 10$ dilutions; (vii) measurement in air; (viii) measurement in PBS $\times 1000$ dilution.

\section{Results with PBS Solutions}

Figure 3(a) shows the transfer characteristics in linear scale at $V_{D}=1 \mathrm{~V}$ measured in air and in different PBS dilutions. The transfer characteristic in air indicates n-type conduction with a threshold voltage, $V_{\mathrm{TH}}$ of $7.7 \mathrm{~V}$. When different PBS dilutions were introduced on the nanowire surface, the n-type behaviour was maintained. However, there was a change in the threshold voltage. The $V_{\mathrm{TH}}$ changed from $11.2 \mathrm{~V}, 13.3 \mathrm{~V}$, and $12.5 \mathrm{~V}$ in $\mathrm{PBS} \times 1000, \mathrm{PBS} \times 100$, and $\mathrm{PBS} \times 10$ solutions, respectively. $I_{\mathrm{ON}}$ is defined as the measured drain current, and $I_{D}$ at $V_{G}=20 \mathrm{~V}$ increased exponentially with decreasing PBS dilutions. The $I_{\mathrm{ON}}$ increased by 9 -fold in PBS $\times 1000$ solution compared to $I_{\mathrm{ON}}$ measured in air. In PBS $\times 100$ solution, $I_{\mathrm{ON}}$ increased by 6.6 times and $I_{\mathrm{ON}}$ escalated by almost 39 times in PBS $\times 10$ solution compared to $I_{\mathrm{ON}}$ measured in air. Since the PBS solutions consist of ions, these ions influenced the surface charges exist on the nanowire surface which then cause the kinks at the transfer curve that vanishes through increasing gate bias.

After each PBS solution was removed, the electrical characteristics were remeasured in air before introducing new solutions. This is important to verify any drift in $I_{\mathrm{ON}}$. Figure 3(b) shows the plot of the drift in $I_{O N}$ against different PBS solutions. $I_{\mathrm{ON}}$ measured in air after removing PBS $\times 1000$ was increased by $39 \%$ from the initial time measured in air before introducing the PBS solution. In case of PBS $\times 100$ solutions, there was a small drift of $12.5 \%$ in $I_{\mathrm{ON}}$ measured before and after dropping the solution. Meanwhile a maximum drift of $169 \%$ in $I_{\mathrm{ON}}$ was shown after the removal of PBS $\times 10$. This large drift in $I_{\mathrm{ON}}$ could be due to the presence of some residues of the $\mathrm{PBS} \times 10$ solution on the $\mathrm{ZnO}$ nanowire surface.

The measured output characteristics, $I_{D}-V_{D}$, are shown in Figure $4(\mathrm{a})$ at a fixed $V_{G}$ of $20 \mathrm{~V}$. The measurements were taken in air and in different PBS solutions. Distinct $n$-type characteristics with a good pinch-off at high drain voltages were observed from the output characteristics. In air, $I_{D}$ measured at $V_{D}=20 \mathrm{~V}$ and $V_{G}=20 \mathrm{~V}$ was $9.5 \mathrm{nA}$; then it increased to $35 \mathrm{nA}$ after PBS $\times 1000$ solution was dropped on the nanowire surface. The current increased further to $48 \mathrm{nA}$ in PBS $\times 100$ solution. Finally, in PBS $\times 10$ solution $I_{D}$ increased by almost 20 times the value measured in air.

Accordingly, the conductances in different measurement conditions were calculated and plotted in Figure 4(b). The 


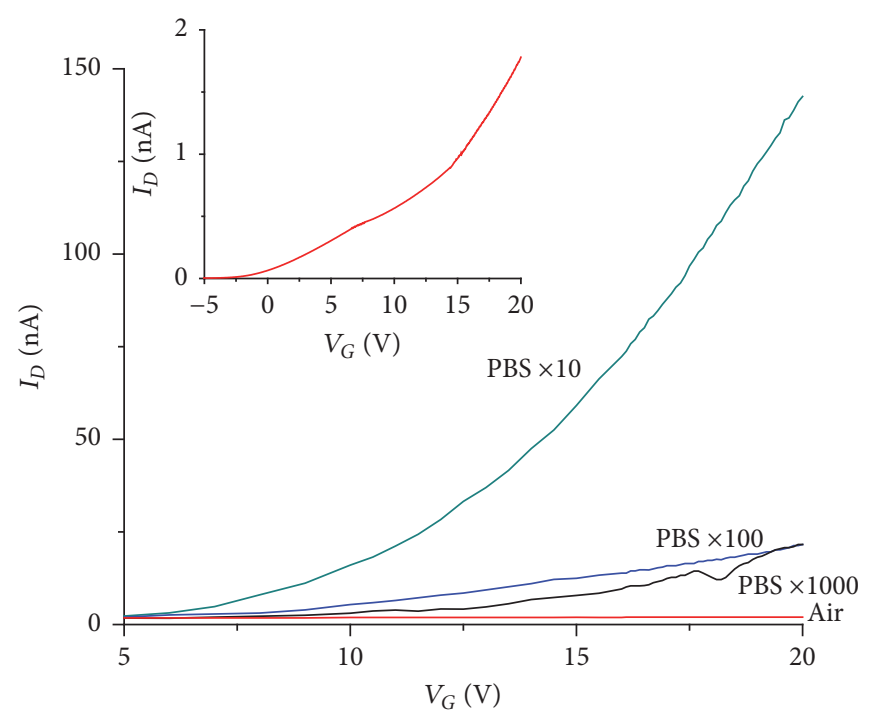

(a)

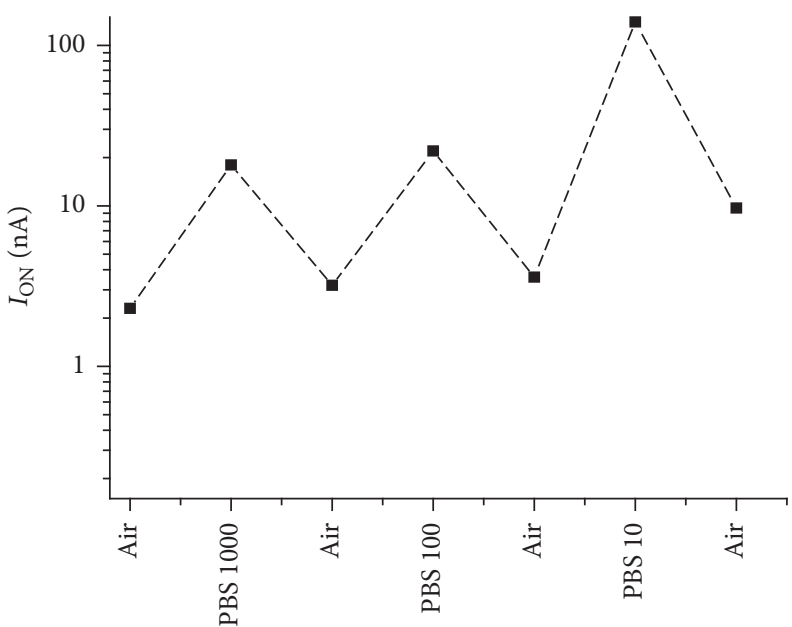

(b)

FIgURE 3: (a) Linear $I_{D}-V_{G}$ plot at $V_{D}=1 \mathrm{~V}$ in air and at different PBS dilutions (inset) enlarged $I_{D}-V_{G}$ plot measured in air. (b) The $I_{\mathrm{ON}}$ measured at $V_{D}=1 \mathrm{~V}$ and $V_{G}=20 \mathrm{~V}$ during liquid and air measurements.

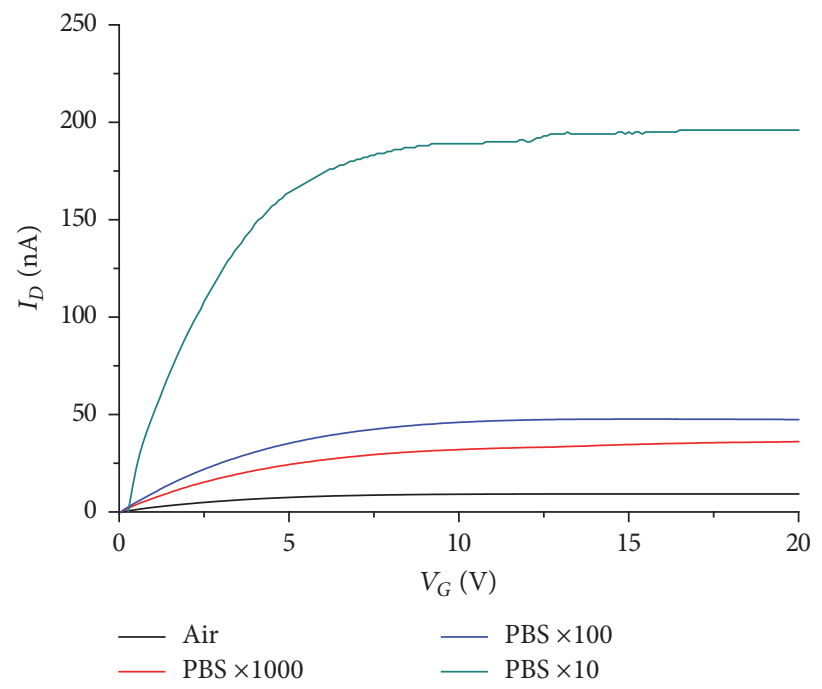

(a)

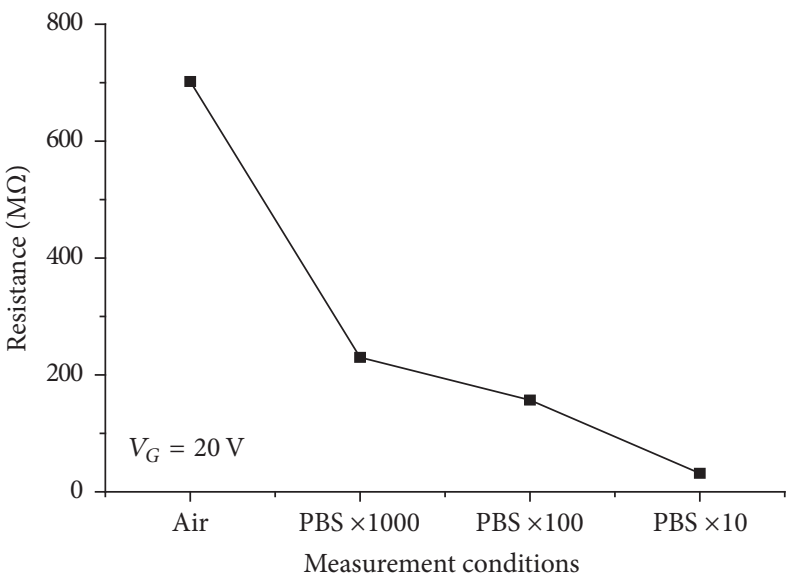

(b)

FIgure 4: (a) The $I_{D}-V_{G}$ at $V_{G}=20 \mathrm{~V}$ measured in air and different PBS solutions. (b) Device resistances extracted from air and liquid measurements.

slopes of the measured $I_{D}-V_{D}$ characteristics measured at $V_{G}=20 \mathrm{~V}$ were calculated at $V_{D}>1 \mathrm{~V}$. The values of resistance were extracted using a linear fit. In air, the resistance measured was found to be the highest at $700 \mathrm{M} \Omega$. The resistances were then decreased from $230 \mathrm{M} \Omega$ in PBS $\times 1000$ to $31 \mathrm{M} \Omega$ in $\mathrm{PBS} \times 10$ solutions.

\section{Discussion}

To date, a high number of ZnO NWFETs commonly operate in atmospheric and vacuum environments, but very few findings on their performance in solutions. In all PBS dilutions, distinct n-type conductions existed despite the exposure of $\mathrm{ZnO}$ nanowires to huge quantity of water molecules and ions. The output characteristics obtained in different PBS dilutions revealed that $\mathrm{n}$-type conductivity was retained with success and could be utilized effectively for biomolecules identification. It is crucial as biosensing practically involves dissolving biological analytes in buffer solutions, with concentration of electrolyte and $\mathrm{pH}$ similar to that of physiological solution. PBS model has a great suitability to serve as a human serum as it exhibits a $7.4 \mathrm{pH}$ [2]. Thus, concentration of electrolyte is a crucial variable of experiment to be taken into account. 


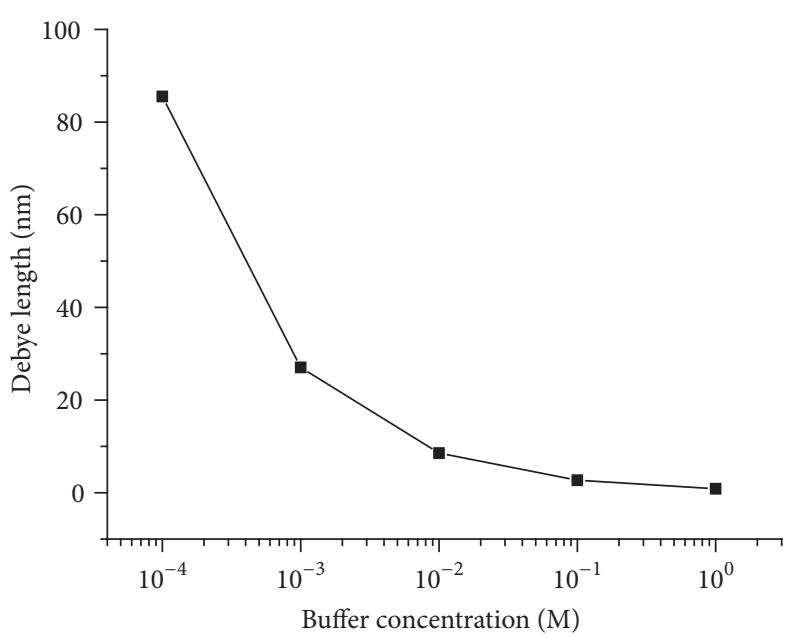

FIGURE 5: Calculated Debye length for various PBS dilutions at $\mathrm{pH}=7.4$.

From computational models of the response time of a nanobiosensor in a diffusion-capture regime, calculations predict that the sensor response varies linearly with $\mathrm{pH}$ and logarithmically with electrolyte concentration [23]. This experimental investigation involved $\mathrm{pH} 7.4$ aqueous PBS, in which the strength of ions degraded with increasing dilutions. The change of $I_{\mathrm{ON}}$, which was defined as the measured drain current, $I_{D}$ at $V_{G}=20 \mathrm{~V}$, was used as the measured response in this experiment. $I_{\mathrm{ON}}$ was found to increase exponentially with decreasing PBS dilutions. Increasing concentrations across all PBS resulted in the production of decreasing $I_{D}-V_{D}$ resistance in a nonlinear way. This revelation is consistent with simulated theoretical studies $[23,24]$ and reported experiment findings in FET [2] and Ion Sensitive FET (ISFET) [25].

Increment of conductance of channel and $I_{\mathrm{ON}}$ alongside the increase of electrolyte concentration is associated with Debye length, $\lambda_{D}$. This $\lambda_{D}$ is defined as the maximum distance at which an external charge can influence the NW carrier concentration [2]. $\lambda_{D}$ is obtained by computing $\lambda_{D}=$ $0.32(I)^{-1 / 2}$, in which $I$ refers to the strength of ions in aqueous buffers. Based on the formula, $\lambda_{D}$ will decrease with an increasing of ion concentration. Figure 5 shows the computed and charted Debye length. This experiment utilized three varieties of aqueous PBS that resulted in three respective $\lambda_{D}$ values, $2.7 \mathrm{~nm}$ for $\times 10(14 \mathrm{mM}), 8.5 \mathrm{~nm}$ for $\times 100(1.4 \mathrm{mM})$, and $27 \mathrm{~nm}$ for $\times 1000(14 \mathrm{mM})$ PBS solutions, respectively. It is observed that little $\lambda_{D}$ produces larger reaction of $I_{\mathrm{ON}}$ in comparison to greater $\lambda_{D}$ in electrolyte solution.

In comparison to $I_{D}$ in PBS solutions, $I_{D}$ in air is much lesser, owing to moisture $\left(\mathrm{OH}^{-}\right)$and oxygen $\left(\mathrm{O}^{2-}\right)$ molecules in air, which depleted the surface of nanowires. In PBS solutions, buffers are formed from phosphate ions. The ions are responsible for neutralization of $\mathrm{OH}^{-}$, which have been discharged by solution's molecules, therefore, maintaining the solution concentration at a constant $\mathrm{pH}$ 7.4. Subsequently, fewer molecules of $\mathrm{OH}^{-}$are available for electron trapping in the surface of nanowires, which resulted in increased $I_{D}$. In PBS $\times 10$ solution, $\lambda_{D}$ exhibited lesser length, indicating effectiveness of charged ions in screening. This screening causes the $\mathrm{OH}^{-}$charged molecule to have little effect on the surface depletion phenomena on the nanowire surface, thus providing an elevating conductance of the device by as much as 39 times $I_{\mathrm{ON}}$ in air. At a lower electrolyte concentration $(\mathrm{PBS} \times 1000)$, the poorly screened $\mathrm{OH}^{-}$ions in the solution will cause a larger depletion layer due to an increase of trapped electrons in the nanowire channel.

The discovery reveals that PBS solutions exhibit passivation characteristic whereby they screen molecules from depleting carriers on nanowire surface (refer to Figure 6). The findings indicate that nanowires fabrication utilizing top-down approach was consistently investigated to explore sensing abilities through biomolecules detection.

\section{Conclusions}

An array of $\mathrm{ZnO}$ NWFET, which consists of 100 nanowires, was successfully fabricated using top-down technique. The device characteristics maintained an $n$-type FET behaviour not only in air but also in different PBS solutions. The device shows higher $I_{\mathrm{ON}}$ in buffer solutions compared to air. In addition, the resistance decreases with increasing electrolyte concentrations. This is due to the passivation characteristic in PBS solutions in which the $\mathrm{OH}^{-}$molecules were effectively screened from the nanowire surface. The effect of PBS solution on the $\mathrm{ZnO}$ nanowire surface suggests that this device is suitable for biosensing applications. In addition, this study indicates that the concentration of PBS used in processes such as biomolecules immobilization will give an effect, which will influence the results obtained. Therefore, the concentration of the PBS solutions used in these processes has to be optimized to achieve accurate results.

\section{Conflicts of Interest}

The authors declare that they have no conflicts of interest.

\section{Acknowledgments}

S. M. Sultan would like to acknowledge the support of the Malaysian Ministry of Higher Education for her postdoctoral study through the MyMentor postdoctoral scholarship, 


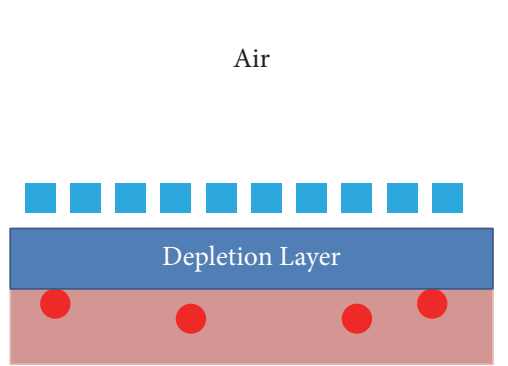

n-type nanowire

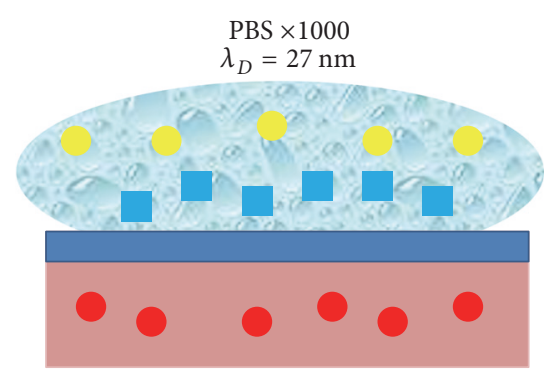

n-type nanowire

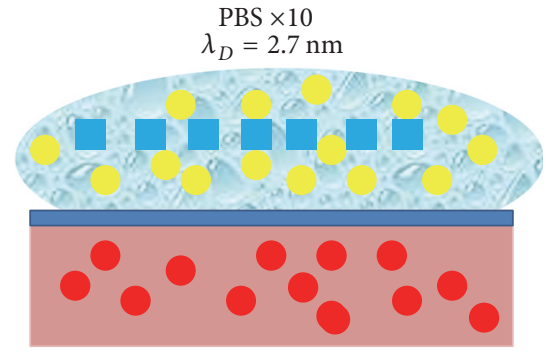

n-type nanowire

Electron carrier

Electron in the buffer

$\mathrm{OH}^{-}$molecule

FIGURE 6: Effect of PBS solutions on the electrical conductance of the ZnO NWFET.

Research University Grant of Universiti Teknologi Malaysia under Projects R.J130000.7823.4F482, and Research University Grant GUP Tier 1, Vote no. 14H63. The authors would like to acknowledge the fabrication and experimental support from the Southampton Nanofabrication Centre.

\section{References}

[1] X. Duan, Y. Huang, Y. Cui, J. Wang, and C. M. Lieber, "Indium phosphide nanowires as building blocks for nanoscale electronic and optoelectronic devices," Nature, vol. 409, pp. 66-69, 2001.

[2] M. Curreli, R. Zhang, F. N. Ishikawa et al., "Label-Free Detection of Biological Entities Using Nanowire-Based FETs," IEEE Transactions on Nanotechnology, vol. 7, no. 6, p. 651, 2008.

[3] K. Chen, B. Li, and Y. Chen, "Silicon nanowire field-effect transistor-based biosensors for biomedical diagnosis and cellular recording investigation," Nano Today, vol. 6, no. 2, pp. 131-154, 2011.

[4] F. Patolsky, G. Zheng, and C. M. Lieber, "Nanowire sensors for medicine and the life sciences," Nanomedicine, vol. 1, no. 1, pp. 51-65, 2006.

[5] X. Liang and S. Y. Chou, "Nanogap Detector Inside Nanofluidic Channel for Fast Real-Time Label-Free DNA Analysis," Nano Letters, vol. 8, no. 5, pp. 1472-1476, 2008.

[6] I. Zeimpekis, K. Sun, C. Hu et al., "Top-down Zinc Oxide Nanowires by Ion Beam Etching for Biosensing Applications," Nanotechnology, vol. 27, 2016.

[7] J. Yuan, R. Duan, H. Yang, X. Luo, and M. Xi, "Detection of serum human epididymis secretory protein 4 in patients with ovarian cancer using a label-free biosensor based on localized surface plasmon resonance," International Journal of Nanomedicine, vol. 7, pp. 2921-2928, 2012.

[8] K. Choi, J. Kim, J. Ahn, J. Choi, M. Im, and Y. Choi, "Integration of field effect transistor-based biosensors with a digital microfluidic device for a lab-on-a-chip application," Lab on a Chip, vol. 12, no. 8, p. 1533, 2012.

[9] X. Duan, Y. Li, N. K. Rajan, D. A. Routenberg, Y. Modis, and M. A. Reed, "Quantification of the affinities and kinetics of protein interactions using silicon nanowire biosensors," Nature Nanotechnology, vol. 7, no. 6, pp. 401-407, 2012.
[10] A. Gao, N. Lu, P. Dai et al., "Silicon-Nanowire-Based CMOSCompatible Field-Effect Transistor Nanosensors for Ultrasensitive Electrical Detection of Nucleic Acids," Nano Letters, vol. 11, no. 9, pp. 3974-3978, 2011.

[11] K. Maehashi and K. Matsumoto, "Label-Free Electrical Detection Using Carbon Nanotube-Based Biosensors," Sensors, vol. 9, no. 7, pp. 5368-5378, 2009.

[12] Y. Ohno, K. Maehashi, and K. Matsumoto, "Chemical and biological sensing applications based on graphene field-effect transistors," Biosensors and Bioelectronics, vol. 26, no. 4, pp. $1727-1730,2010$.

[13] S. M. Sultan, K. Sun, O. D. Clark et al., "Electrical Characteristics of Top-Down ZnO Nanowire Transistors Using Remote Plasma ALD," IEEE Electron Device Letters, vol. 33, no. 2, pp. 203-205, 2012.

[14] Z. Fan, D. Wang, P.-C. Chang, W. Tseng, and J. G. Lu, "ZnO nanowire field-effect transistor and oxygen sensing property," Applied Physics Letters, vol. 85, no. 24, Article 5923, 2004.

[15] Z. F. Z. Fan and J. G. Lu, "Chemical sensing with $\mathrm{ZnO}$ nanowires," IEEE Transactions on Nanotechnology, vol. 5, no. 4, p. 393, 2006.

[16] S. K. Arya, S. Saha, J. E. Ramirez-Vick, V. Gupta, S. Bhansali, and S. P. Singh, "Recent advances in $\mathrm{ZnO}$ nanostructures and thin films for biosensor applications: review," Analytica Chimica Acta, vol. 737, pp. 1-21, 2012.

[17] Z. Zhao, W. Lei, X. Zhang, B. Wang, and H. Jiang, "ZnO-Based Amperometric Enzyme Biosensors," Sensors, vol. 10, no. 2, pp. 1216-1231, 2010.

[18] X. Wang, F. Yang, W. Yang, and X. Yang, "A study on the antibacterial activity of one-dimensional $\mathrm{ZnO}$ nanowire arrays: effects of the orientation and plane surface," Chemical Communications, no. 42, p. 4419, 2007.

[19] G. Mo, "Unusual electrochemical response of $\mathrm{ZnO}$ nanowiresdecorated multiwalled carbon nanotubes," Electrochim Acta, vol. 55 , no. 2, p. 511, 2009.

[20] S. M. Sultan, Top-down fabrication and characterization of zinc oxide nanowire field effect transistors [Ph.D. Thesis], University of Southampton, Southampton, England, 2013.

[21] M. M. Hakim, M. Lombardini, K. Sun et al., "Thin Film Polycrystalline Silicon Nanowire Biosensors," Nano Letters, vol. 12, no. 4, pp. 1868-1872, 2012. 
[22] S. M. Sultan, K. Sun, M. R. de Planque, P. Ashburn, and H. M. Chong, "Top-down fabricated $\mathrm{ZnO}$ nanowire transistors for application in biosensors," in Proceedings of the ESSDERC 2012 42nd European Solid State Device Research Conference, pp. 137140, Bordeaux, France, September 2012.

[23] P. R. Nair and M. A. Alam, "Screening-Limited Response of NanoBiosensors," Nano Letters, vol. 8, no. 5, pp. 1281-1285, 2008.

[24] D. Song, T. J. Pelura, J. Liu, and Y. Ni, "Effects of buffer $\mathrm{pH}$ and phosphate concentration on the droplet size and EYP hydrolysis of perflubron/EYP emulsions," Artificial Cells, Blood Substitutes, and Immobilization Biotechnology, vol. 22, no. 4, p. 1299, 1994.

[25] C. S. Weng, U. Hashim, and W. Liu, "The effect of phosphate buffer solution (PBS) concentration on the ion sensitive fieldeffect transistor (ISFET) detection," in Proceedings of the 2013 IEEE Regional Symposium on Micro and Nanoelectronics (RSM), pp. 200-203, Daerah Langkawi, Malaysia, September 2013. 

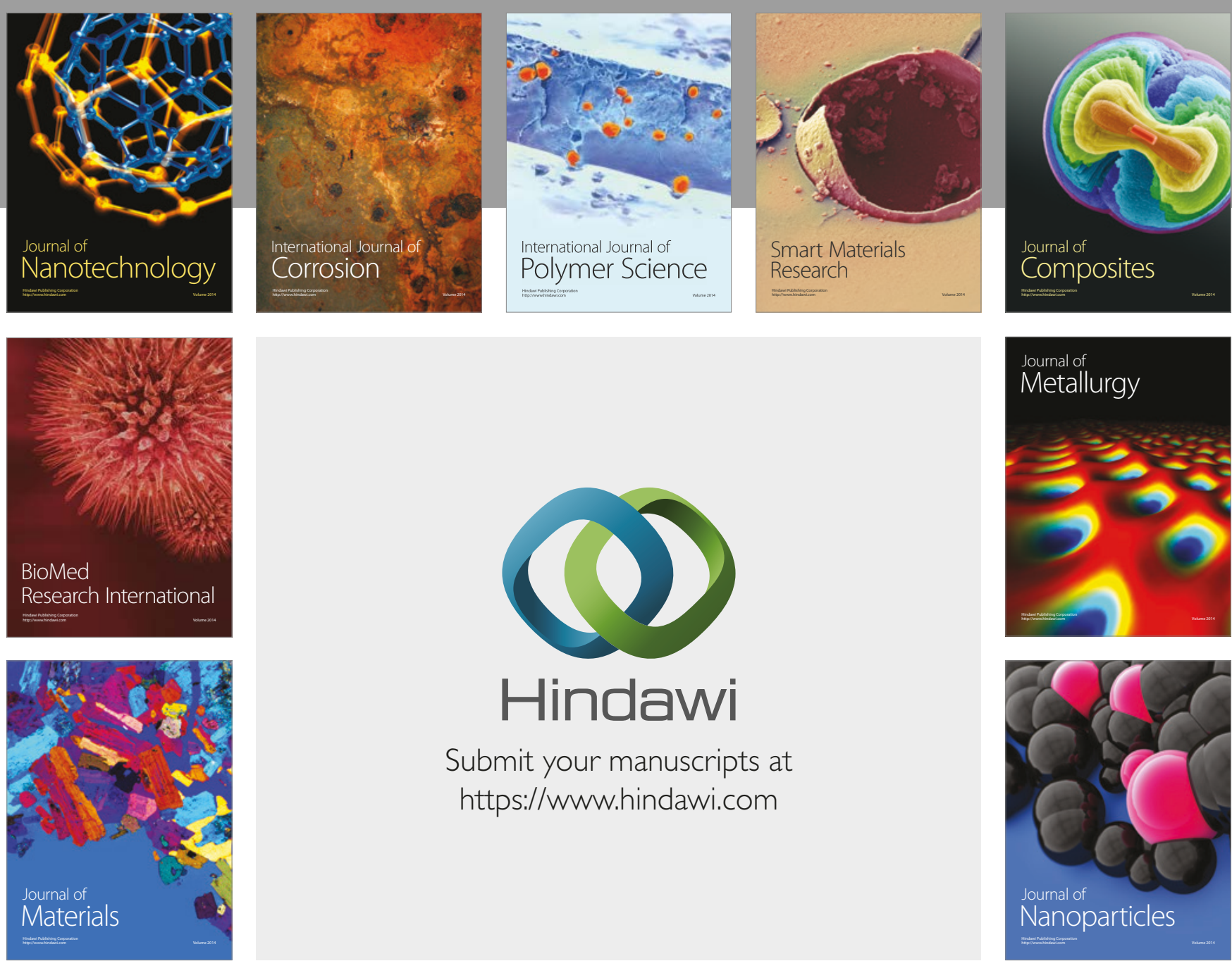

\section{Hindawi}

Submit your manuscripts at

https://www.hindawi.com
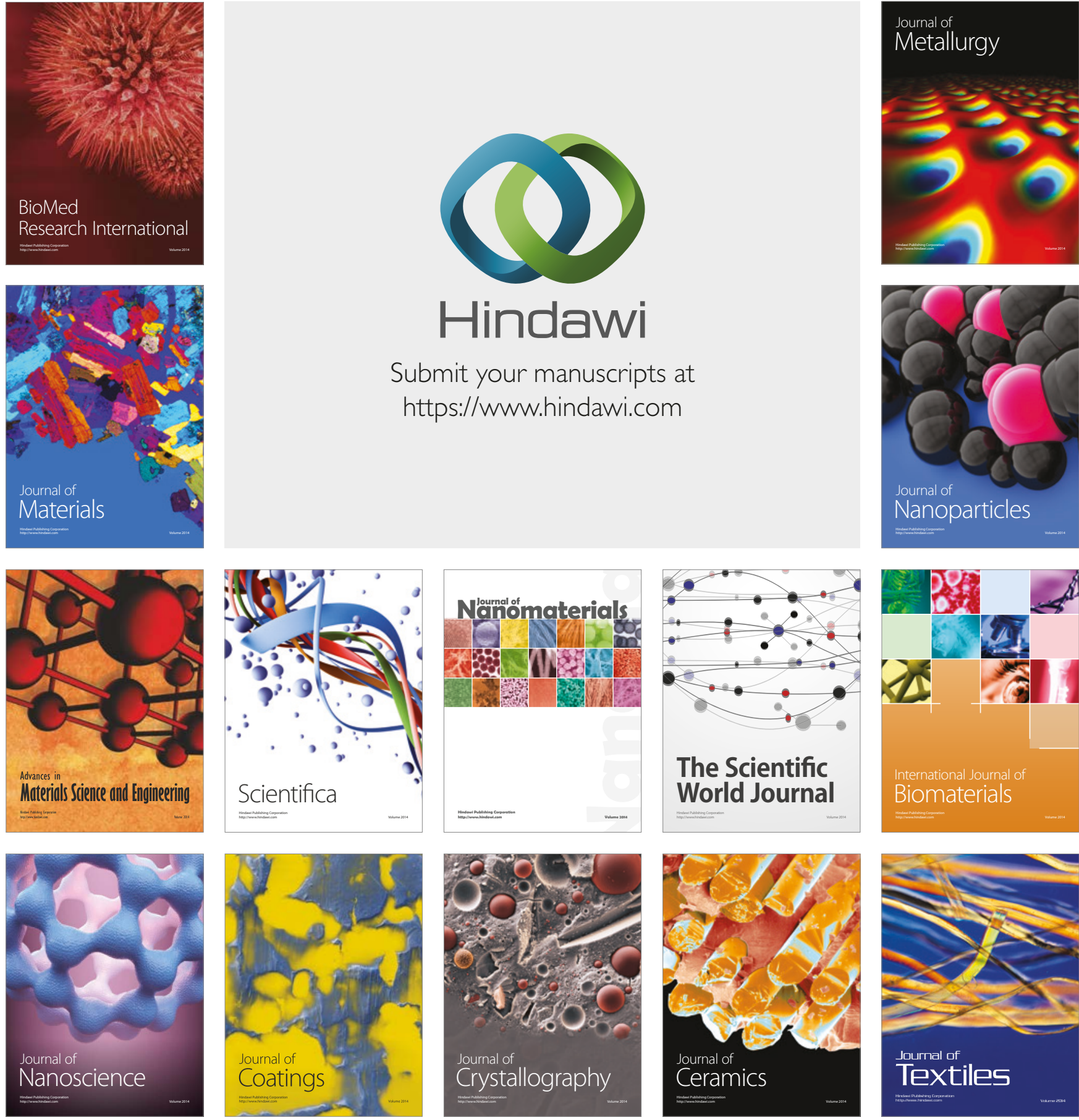

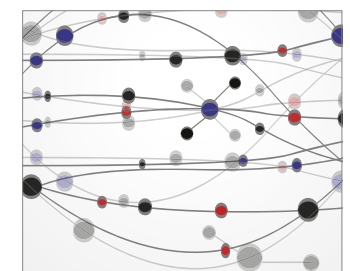

The Scientific World Journal
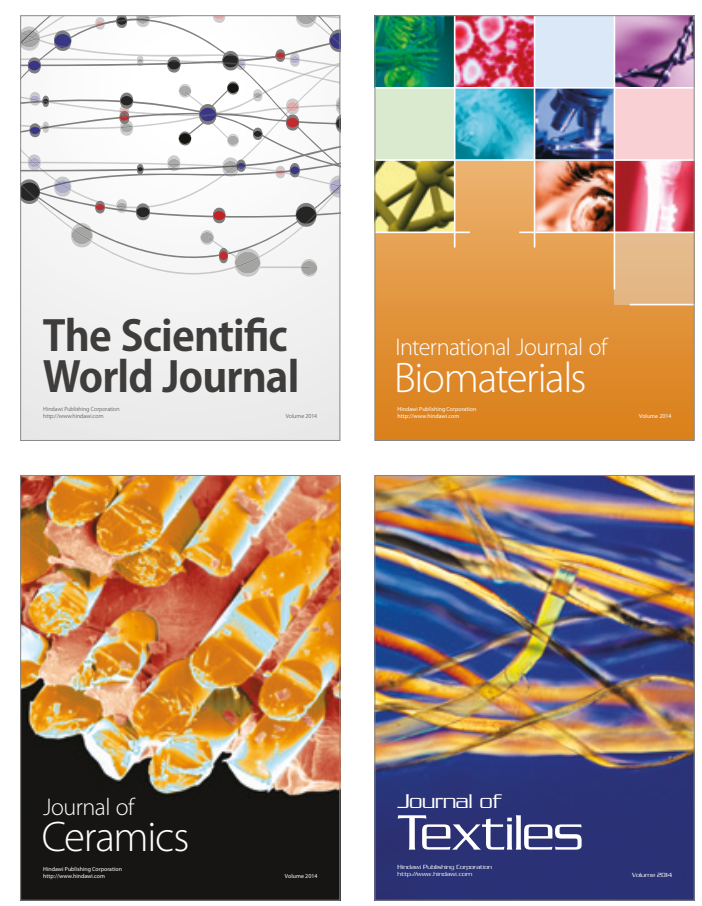